Onkologe 2022 28 (Suppl 1):S4-S5

https://doi.org/10.1007/s00761-022-01104-z Angenommen: 20. Januar 2022

(๑) The Author(s), under exclusive licence to Springer Medizin Verlag $\mathrm{GmbH}$, ein Teil von Springer Nature 2022

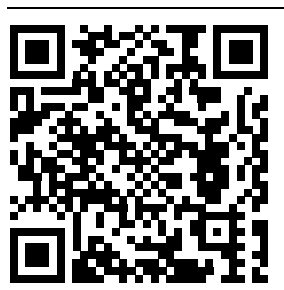

QR-Code scannen \& Beitrag online lesen

\title{
Die Kooperation des Berufsverbands der Niedergelassenen Hämatologen und Onkologen (BNHO) mit der AIO
}

\author{
Neue gemeinsame Wege für eine erfolgreiche klinische \\ Forschung in der Onkologie
}

\author{
Wolfgang Knauf' · Anke Reinacher-Schick ${ }^{2}$ Thomas IIImer ${ }^{3}$ Eray Gökkurt ${ }^{4}$ \\ 'Onkologische Gemeinschaftspraxis, Frankfurt am Main, Deutschland \\ ${ }^{2}$ St. Josef-Hospital, Klinik für Hämatologie und Onkologie mit Palliativmedizin, Klinikum der Ruhr- \\ Universität, Bochum, Deutschland \\ ${ }^{3}$ Gemeinschaftspraxis Hämatologie - Onkologie, Dresden, Deutschland \\ ${ }^{4}$ Hämatologisch-Onkologische Praxis Eppendorf (HOPE), Hamburg, Deutschland
}

Der Berufsverband der Niedergelassenen Hämatologen/Onkologen in Deutschland e. V. (BNHO) tritt für die berufspolitischen Belange seiner aktuell rund 600 Mitglieder ein. Diese betreuen pro Quartal etwa 500.000 Patienten mit einer hämatologischen oder onkologischen Diagnose. Einer langen Tradition folgend engagieren sich die Mitglieder des BNHO in verschiedenen Studiengruppen wie auch in der Arbeitsgemeinschaft Internistische Onkologie (AIO). So tragen die Praxen regelhaft zum erfolgreichen Abschluss sowohl von Zulassungsstudien neuer innovativer Substanzen als auch von Therapieoptimierungsstudien bei. Eine umfängliche Berufspolitik ist heutzutage nicht mehr ohne wissenschaftspolitische Aktivitäten denkbar. Dies ist der Ansatzpunkt, die in der Vergangenheit sich eher in Einzelaktionen manifestierende Zusammenarbeit des BNHO mit der AIO neu zu strukturieren.

Immer mehr in den Vordergrund nicht nur der differenzialdiagnostischen $\mathrm{Ab}$ grenzung, sondern auch der therapeutischen Stratifizierung sind immunphänotypische (z.B. PD-L1-Status) und molekularpathologische Befunde (z.B. EGFR-Mutations-Status) getreten. Diese ermöglichen den rationalen Einsatz vom Immunthera- peutika und zielgerichteter Substanzen. Damit verbunden ist die Notwendigkeit nicht nur zur kritischen Auswahl der Medikamente; die zumeist hochpreisigen Therapien bedürfen einer Überprüfung von Sicherheit und Effektivität an Patientenkollektiven, die für statistische Auswertungen geeignet erscheinen. Damit rückt die Versorgung in den Blick der wissenschaftlichen Evaluation und ermöglicht die Generierung neuer Fragestellungen. Aus der Versorgung heraus entstehen Impulse für Grundlagenforschung und Medikamentenentwicklung.

Die "Nationale Dekade gegen Krebs", eine gemeinsame Initiative von BMBF und $B M G$, fordert und fördert folgerichtig die Vernetzung von Forschungsanstrengungen mit der Umsetzung im Praxisalltag. Durch das strukturierte Sammeln und die Analyse der Praxiserfahrungen entsteht eine "Wissen generierende Versorgung" als Grundlage für die Sicherung von Qualität und Ausgangspunkt für neue Therapieprinzipien.

Schon lange wurden in die Studien der AIO Patienten von niedergelassenen internistischen Onkologen (NIO) eingebracht und haben sich NIO in den Arbeitsgruppen der AIO engagiert. Diese bewährte 
Zusammenarbeit wird nun strukturell ergänzt durch die Gründung einer gemeinsamen Forschungsplattform (CONNECT, siehe Hauptartikel) innerhalb der erweiterten Arbeitsgruppe „molekulare und translationale Onkologie“. Hier sollen u.a. gemeinsame Datenbanken installiert werden vor dem Hintergrund der Tatsache, dass viele onkologisch erkrankte Patienten zwischen stationären und ambulanten Therapiephasen wechseln, sinnvollerweise aber der Gesamtverlauf erfasst werden sollte.

》) Onkologie wird aus der Sicht des Patienten von der Diagnose bis hin zur Nachsorge integrativ gedacht

Damit entsteht eine neue gesundheitspolitische Perspektive: Die sektorale Datenerfassung und Sichtweise auf die Erkrankung und die Konzentration auf einzelne Krankheitsphasen mit spezifischen Interventionen wird verlassen, Onkologie wird aus der Sicht des Patienten von der Diagnose bis hin zur Nachsorge integrativ gedacht.

Der Anteil der NIO unter den AIO-Mitgliedern beträgt ca. $25 \%$. Die NIO stellen in den Studien, bei denen die AIO-Studien-GmbH als Sponsor tätig ist, konstant fast $40 \%$ aller Prüfzentren. Der größte Teil der NIO sind im BNHO organisiert. Mit der Kooptierung von PD Dr. med. Thomas IIImer als BNHO-Vertreter in den Vorstand der AIO sollen die vielfältigen Anknüpfungspunkte zwischen der AIO und dem $\mathrm{BNHO}$ intensiviert und gefördert werden. Ziel ist es, die Expertise besonders in der flächendeckenden, lokalen Umsetzung der onkologischen Versorgung in die AIO-Projekte einfließen zu lassen, aber auch die Rekrutierungsleistung der NIO in den AIOStudien zu steigern. Die geplante Kommunikationsebene soll die Kontaktaufnahme und den Dialog zwischen interessierten $\mathrm{NIO}$ und den AlO-Arbeitsgruppen vereinfachen und fördern.

In der Kooperation von BNHO und $\mathrm{AlO}$ können über Sektorengrenzen hinweg onkologische Fragestellungen mit unmittelbarem Praxisbezug an großen Patientenkollektiven bearbeitet werden. Dies geht nicht nur einher mit der Implementierung neuer Therapien, sondern dient in besonderem Maße der Qualitätssicherung in der onkologischen Versorgung.
Prof. Dr. Wolfgang Knauf

Vorsitzender Berufsverband der Niedergelassenen Hämatologen \& Onkologen in Deutschland e.V. (BNHO)

Prof. Dr. Anke Reinacher-Schick

Vorsitzende der Arbeitsgemeinschaft Internistische Onkologie (AIO) in der Deutschen Krebsgesellschaft e. V. (DKG)

Korrespondenzadresse

Prof. Dr. Wolfgang Knauf

Onkologische Gemeinschaftspraxis

Im Prüfling 17-19, 60389 Frankfurt am Main, Deutschland

info@onkologie-bethanien.de

\section{Prof. Dr. Anke Reinacher-Schick}

St. Josef-Hospital, Klinik für Hämatologie und Onkologie mit Palliativmedizin, Klinikum der Ruhr-Universität

Gudrunstraße 56, 44791 Bochum, Deutschland reinacher@aio-portal.de

Interessenkonflikt. W. Knauf, A. Reinacher-Schick, T. Illmer und E. Gökkurt geben an, dass kein Interessenkonflikt besteht.
Hier steht eine Anzeige. Springer 